\title{
Efficacy of Neuropsychological Rehabilitation Applied for Patients With Bipolar Disorder ${ }^{*}$
}

\author{
Fabricia Quintão Loschiavo-Alvares \\ Nexus Neuropsychology Clinic, \\ Minas Gerais, Brazil
}

\author{
Fernando Silva Neves \\ Universidade Federal de Minas Gerais, \\ Belo Horizonte, Brazil
}

\begin{abstract}
Objective: The aim of the present study was to assess the efficacy of neuropsychological rehabilitation on executive functions, quality of life, and coping strategies in a sample of euthymic patients with BD (bipolar disorder) type I and type II. Method: It was a quasi experimental and longitudinal study which enrolled 50 outpatients with DSM-IV BD. NR (neuropsychological rehabilitation) plus pharmacotherapy group $(\mathrm{NRG}=20)$ was compared with pharmacotherapy-control group $(\mathrm{CG}=30)$ over 14 weeks. The NR protocol was divided into three modules. The first was directed toward mood monitoring, the following focused on executive functions, and the third addressed attention and memory rehabilitation. As outcome measures, it was used an ecological questionnaire of executive functions, DEX-R, quality of life indicators provided by Whoqol-Bref and coping through Ways of Coping Scale. It was also assessed clinical variables, such as frequence of manic, hypomanic and depression episodes. Results: Mixed-design ANOVA (Analysis of Variance) demonstrated significant improvements in all measures applied from baseline to endpoint as well as after the conclusion of NR protocol (six months) in NRG, suggesting an interaction between treatment and time. The reliable change index in NRG ranged from $45 \%$ to $90 \%$ for all outcome measures. Cohen's $d$ for the differences between the mean scores of pre- and post-treatment for the two groups revealed very robust effect size for the NRG $(d>0.80)$. Conclusions: The results showed greater improvements for the NRG, highlighting the efficacy of neuropsychological rehabilitation and its superiority as well, when it was compared with treatment as usual, which was pharmacotherapy only.
\end{abstract}

Keywords: neuropsychological rehabilitation, cognitive rehabilitation, cognition, BD (bipolar disorder), dex, coping, quality of life

\section{Introduction}

BD (Bipolar disorder) is well recognized as a chronic illness with substantial psychosocial and occupational morbidity, according to Bearden, Hoffman, and Cannon (2001). Over the past decade, a number of studies have identified cognitive deficits in $\mathrm{BD}$ as an important determinant of this disability, as shown in Bowie et al. (2010). Robinson, Thompson, Gallagher, Goswami, Young, and Ferrier (2006) and Robinson and Ferrier (2006) described these deficits persist in the absence of acute symptoms and seems to worsen

\footnotetext{
*Acknowledgements: This paper is supported by Grant INCT-MM (FAPEMIG: CBB-APQ-00075-09 / CNPq 573646/2008-2). Fabricia Quintão Loschiavo-Alvares, Ph.D., Nexus Neuropsychology Clinic, fabricia@nexusclinica.com.br. Fernando Silva Neves, Ph.D., Faculdade de Medicina, Universidade Federal de Minas Gerais.
} 
with cumulative episodes. Neuropsychological deficits have been recognized as one core feature of BD due to their prominent nature and debilitating functional consequences, as pointed recently by Harvey (2011). Recent studies conducted by Robinson et al. (2006) and Robinson and Ferrier (2006) have suggested medium to large effect sizes for the neuropsychological differences between people with BD and control subjects, especially in the domains of episodic memory, attention/concentration, and executive functioning. Martinez-Arán et al. (2007) have demonstrated that the main cognitive impairment is related to executive functions, while moderate cognitive deficits was observed in other domains, such as verbal memory, response inhibition, sustained attention, psychomotor speed, abstraction, and set-shifting. Efforts to improve the prognosis and functional outcomes in $\mathrm{BD}$ have prompted the interest and development of neuropsychological rehabilitation programmes. These comprise neurocognitive techniques, training psychoeducation on cognitive-related issues as well as problem-solving within an ecological framework, as proposed by Matrinez-Aran et al. (2011), having as an ultimate goal the improvement of everyday functioning.

To the best of our knowledge, until recently, there were two studies which investigated the neuropsychological rehabilitation in BD. Deckerbasch et al. (2010) explored whether a new cognitive remediation treatment designed to treat residual depressive symptoms as well as addressed to cognitive impairment would be associated with improvement in psychosocial functioning in individuals with BD. It was found that 18 individuals with BD both at the end of treatment, as well as at the 3-month follow-up, showed lower residual depressive symptoms, and increased occupational, as well as overall psychosocial functioning. These findings have suggested that treating residual depressive symptoms and cognitive impairment may be an avenue to improving occupational and overall functioning in individuals with BD. Torrent et al. (2013), in a recent investigation, sought to assess the efficacy of functional remediation on functional improvement in a sample composed by euthymic patients with BD. This was a multicenter, randomized, rater-blind clinical trial involving 239 outpatients. The sample was divided into three groups: functional remediation $(n=77)$, psychoeducation $(n=82)$, and usual treatment $(n=80)$ over 21 weeks. The results have shown that functional remediation differed significantly from treatment as usual, but not from psychoeducation.

The aim of the current study was to assess specifically the efficacy of NR (neuropsychological rehabilitation) for bipolar patients, which comprised a set of neurocognitive techniques and strategies addressed to attention, memory, and executive functions having as a final goal the optimisation of functional capacities. Based on the literature, it was hypothesised that the patients in the NR group would have greater improvement in quality of life, coping skills, and cognitive functions, when compared with the another group under pharmacotherapy only.

\section{Methods}

\section{Study Design and Sample}

The research conducted was a quasi experimental and longitudinal study, which aimed to investigate the effect of a protocol of NR in $\mathrm{BD}$, and so it was used a pseudo random allocation between groups, which were control passive group-CG (pharmacotherapy only without any adjunctive psychosocial therapy) and intervention group - NRG (pharmacotherapy plus NR treatment). The pharmacological treatment was prescribed according to local guidelines for the best management of BDs, and this treatment was kept stable in 
two groups. The patients were assessed with the efficacy measures adopted at the beginning of the protocol, after its completion (14 weeks) and after six months of follow-up (withdrawn phase).

\section{Participants}

The current study included 50 patients with diagnoses of bipolar I or II disorder according to DSM-IV criteria (see Figure 1), so it was used a semi structured interview-MINI (Mini International Neuropsychiatric Interview) proposed by Amorim (2000). All the participants were recruited mainly via local advertising and information distributed on a psychiatric ambulatory at university campus. All patients were tested with a comprehensive neuropsychological battery addressed to assess memory, attention, and executive functions. To evaluate verbal memory, RAVLT (the Rey Auditory Verbal Learning Test) (Malloy-Diniz, Lasmar, Gazinelli, Fuentes, \& Salgado, 2007) was used. The Rey Complex Figure was used for evaluation of planning and non-verbal memory. CPT-II (the Continuous Performance Test) (Conners, 2003) was used to evaluate attention. For executive functions, IGT (the Iowa Gambling Task) (Malloy-Diniz, Leite, Moraes, Correa, Bechara, \& Fuentes, 2008) and WCST (Wisconsin Card Sorting Test) were used. Finally, to evaluate impulsivity, BIS-11 (the Barratt Impulsivity Scale) was applied.

After this assessment, the patients were stratified to particular groups, being NRG or CG. The NR program was part of ongoing psychiatric outpatient treatment at Clinical Hospital, in Belo Horizonte, Brazil. It was required to be part of this study at least three months of clinical remission, be euthymic as well as taking the same medication for at least three months. To best define euthymia, it was used Young Mania Rating Scale (Young, Biggs, Ziegler, \& Meyer, 1978) score $<10$ and Beck Depression Inventory (Beck, Steer, \& Garbin, 1988) score $<10$. Exclusion criteria adopted were any medical condition that could affect neuropsychological performance (such as neurological diseases), substance abuse or dependence, ECT (eletroconvulsive therapy), participating in any structured psychological intervention, such as psychotherapy or psychoeducation, any co morbid psychiatric condition and any medication changes during the study. This research was approved by an ethic committee and all patients provided written informed consent before take part of this study.

\section{Neuropsychological Rehabilitation Intervention}

Firstly, it was developed a pilot study to best define the protocol of NR (Loschiavo-Alvares et al., 2013). It was conducted an investigation using a single case design. The protocol proposed was based on the research conducted by Deckersbach et al. (2010). It was applied and has shown to be successful in improving the patient's functional ability toward the level of performance shown by six matched controls. After this pilot, in whom the feasibility of the protocol was demonstrated, this was adopted in this current investigation. It is important to highlight that only one therapist worked with all patients.

The NR protocol comprises 14 weekly individual sessions (50 minutes each) divided into three modules and each of them containing four sessions, so the whole protocol lasted three and half months (see Table 1). During all modules and respective sessions, all strategies were applied in a tailored way, focused on patients' functional needs considering their occupations. However, the same strategies addressed for attention, memory, and executive functions were applied for all patients. The content of this intervention was based on ecological tasks which were required to be performed in the clinical setting, as well as at home (Torrent et al., 2013). The techniques and strategies adopted were proposed in accordance with consolidated programs for NR suggested by Wilson (2005) and Solhberg and Mateer (2001) and most of them were paper and pencil tasks. 


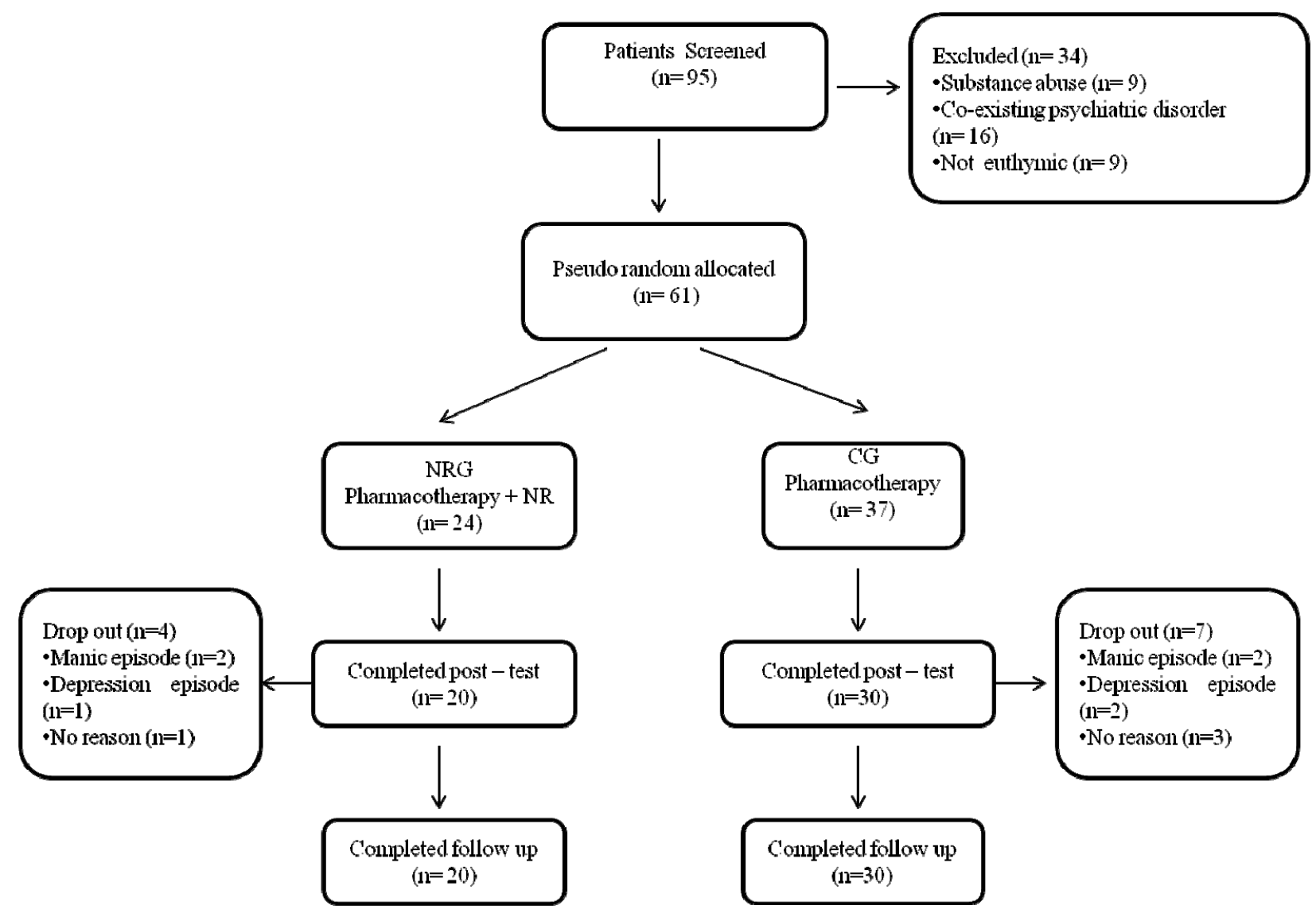

Figure 1. Flowchart of patient participation.

Table 1

NR Protocol

\begin{tabular}{lll}
\hline Modules & Sessions & Sessions content \\
\hline- & 1 & Presentation of the protocol and establishment of functional measures \\
& 2 & What is BD? Illness awareness and lifestyle regularity \\
Module 1: Psychoeducation & 3 & Pharmacotherapy and BD \\
& 4 & Cognition and BD \\
& 5 & My symptoms-mood chart \\
Module 2: Rehabilitation of & 7 & What is attention and strategies to improve it \\
attention and memory & 8 & Daily life application of attention strategies \\
& 9 & What is memory and strategies to improve it; agenda and external devices \\
& 10 & Daily life application of memory strategies \\
Module 3: Rehabilitation of & 11 & Self-instructions and self-monitoring. Goal management training \\
executive functions & 12 & How to program and organize tasks: priorities, tasks segmentation, time management, \\
& & feedback, checklists \\
& 13 & Problem-solving techniques \\
& 14 & Daily life application of executive functions strategies \\
& & Continuous use of learned techniques and acquired competences
\end{tabular}

\section{Assessments}

Innitially, the sociodemographic data were collected, such as age, sex, education level, occupational status, 
illness duration, age at illness onset, and number of hospitalizations. Many efficacy measures were used: DEX-R, Whoqol-Bref and Ways of Coping Scale, all self-reported instruments, which were described below. These assessments were conducted at the beginning as well as at the end of the intervention, and also after six months of the completion of the NR protocol.

DEX-R. The DEX-R questionnaire consists of a checklist of symptoms on a 5-point Likert scale, ranging from (0) "Never" to (4) "Very often". This questionnaire is available in two different versions, being self-reported, used in this study, and an independent rating one. Initially, this questionnaire was proposed as a supplement for the primary tests of BADS (Behavioural Assessment of the Dysexecutive Syndrome), elaborated by Wilson, Alderman, Burgess, Emslie, and Evans (1996). It was designed to screen for changes in observable everyday manifestations of executive and cognitive dysfunction following acquired brain injury. The DEX encompassed 20 items that aimed to evaluate abstraction, impulsivity, confabulation, planning skills, mood regulation, and decision-making, and it covers a wide range of specific problems such as difficulties with attention, memory, information processing, behavioural control, emotion regulation and awareness as reported by Simblett and Bateman (2011). Even though the DEX was originally designed for use as qualitative assessment for focusing rehabilitation on an individual's personal difficulties, this tool is currently used as a quantitative test instrument for diagnoses purposes, for example, Bennett and Ponsford (2005) and Perez et al. (2009). In a recent investigation, Loschiavo-Alvares et al. (2013) have found three dimensions for a sample with $\mathrm{BD}(n=120)$. The factors which loaded in three main dimensions were congruent with the functions that have been attributed to orbitofrontal (OFC - social-regulation), dorsolateral (DL-flexibility, fluency, and working memory), and anterior cingulated ( $\mathrm{CA}$-motivation and attention) circuits. In that way, the former dimension was composed by functions related to social skills and self-regulation, the second one by motivation and attention functions and the last dimension was comprised by flexibility, fluency, and working memory.

Ways of Coping Scale. The Ways of Coping Scale adapted by Seidl, Tróccoli, and Zannon (2001), comprises four factors: coping strategies based on the problems, coping strategies based on the emotion, religiosity/fantasy thinking, and search for social support.

Coping, as pointed by Lazarus and Folkman (1984), can be defined as thoughts and behaviors that individuals use to manage the internal and external demands of situations that are appraised as stressful. Coping strategies in those with BD have long been investigated. Greenhouse et al. (2000) have highlighted that coping strategies focused on low levels of acceptance and high levels of denial have been linked with poor medication adherence in bipolar I disorder, while maladaptive coping strategies, such as rumination about negative states, as shown in Nolen-Hoeksema (2000), are linked to increased depression. In accordance to those data, encouraging the development of a more adaptative coping strategies is one of the central components of a cognitive intervention in patients with BD, as suggested by Kapczinski et al. (2008) and Grassi-Oliveira, Daruy-Filho, and Brietzke (2010). Based on this assumption, the coping was used in this study as a measure of efficacy of the NR protocol.

WHOQOL-Bref Portuguese version. The WHOQOL-BREF is a self-administered, multidimensional questionnaire with 26 items containing four domains: physical health (questions related to pain, sleep and energy, treatment adherence, and daily activities), psychological (questions related to positive and negative feelings, self-esteem, personal beliefs, attention and concentration, and body image), social relationships (addresses to personal relationships, social support, and sexual activity), and environmental (encompasses questions related to security, financial support, health and social care, access to knowledge, participation and leisure). Each 
domain, in accordance with the WHOQOL Group (2000), includes three to eight items. The first item (Q1) asks the overall quality of life, and the second item (Q2) asks questions on general health satisfaction. Each item is based upon a self-report using a 5-point scale, which is applied in all questions. Respondents then check one of the following degrees: "Not at all", "A little", "Moderately", "Mostly", and "Completely" (Fleck et al., 2000). Amini and Sharifi (2012) have already applied this questionnaire to assess quality of life in BD patients.

Data analyses. All statistical analyses were conducted using SPSS (the Statistical Package for Social Sciences) Version 20.0. As the first step, descriptive analyses were conducted. Then Mixed-Design ANOVA (Analysis of Variance) $3 \times 2$ was used to examine the treatment effect (pre- $\times$ post-NR protocol $\times$ follow-up) considering the two groups (NRG X CG). In all cases which the Mauchly W's test indicated that the sphericity assumption was violated, the statistics were reported with the Greenhouse-Geisser correction. Furthermore, to explore the effect of the intervention on scores, the effect size within groups was calculated ( $d$ Cohen) considering the pre- and post-treatment means.

We used an RCI (reliable change index) in accordance with Jacobson and Truax (1991) to determine the proportion of participants who showed treatment improvements from pre- to post-treatment and from pre-treatment to long-term follow-up. Individual-level change scores were examined in two ways. First, the standard deviation method was used, calculated as $(\mathrm{X} 2-\mathrm{X} 1) / S D$, where $\mathrm{X} 1$ and $\mathrm{X} 2$ are the individual's observed pre-treatment and post-treatment as well as pre-treatment and long-term six-month follow-up scores. Corrections for measurement error and practice effects were then calculated for each participant using the RCI, calculated as $([\mathrm{X} 2-\mathrm{X} 1]-[\mathrm{M} 2-\mathrm{M} 1]) / S D$, where $\mathrm{X} 1$ and $\mathrm{X} 2$ are the individual's observed pre-treatment and post-treatment or long-term six-month follow-up scores, M1 and M2 are the group mean pre-treatment and post-treatment or long-term six-month follow-up scores, and $S D$ is the standard deviation of the group pre-treatment-post-treatment and pre-treatment-follow-up difference score.

For each of the two methods, reliable change was defined both (1) as a $1 S D$ change in performance, and (2) with $\alpha$ set at 0.10 (two-tailed; 90\% confidence of improvement), which requires a more conservative 1.645 $S D$ change in performance.

Finally, it was conducted $t$-test to compare the mean of mood swings, hypo/manic and depression episodes between groups.

\section{Results}

Sample demographics were compared across both groups. There were no significant differences on age, gender, education level, estimated IQ, BD types, illness duration, age at illness onset, and number of hospitalizations (all $p s>0.10$ ). It suggests that the pseudo-randomization process was efficient. Demographic data are summarized in Table 2. During the intervention, $16.6 \%$ and $18.9 \%$ of the patients discontinued in the NRG and CG respectively. There were no significantly differences between groups.

\section{DEX-R}

Mixed-model repeated measures analysis indicated a significant interaction between the treatment and time for all the three subscales (OFC, CA, and DL), as well as for the total score (OFC $=F[2,98]=17.54, p<$ $0.001, \eta_{p}^{2}=0.26 ; \mathrm{CA}=F[2,9]=55.91, p<0.001, \eta_{p}^{2}=0.53 ; \mathrm{DL}=F[1,49]=51.08, p<0.001, \eta_{p}^{2}=0.08$; Total $=F[2,98]=11.82, p<0.001, \eta_{p}^{2}=0.19$ ). These results showed that the performance in DEX-R over the three assessments differed between CG and NRG. 
The contrast measure for groups difference regarding pre- and post-treatment was significant $(\mathrm{OFC}=F[1$, $49]=20.74, p<0.001 ; \mathrm{CA}=F[1,49]=39.15, p<0.001, \eta_{p}^{2}=0.44 ; \mathrm{DL}=F[1,49]=5.51, p=0.02, \eta_{p}^{2}=$ 0.10 ; Total $=F[1,49]=34.54, p<0.001$ ), which means that changes in scores, in other words, the important decrease of these, was greater for the group under NR intervention.

On the other hand, the contrast measure for the difference between post-treatment and follow-up scores, when compared groups, considering OFC subscale was not significant $(F[1,49]=0.02, p=0.89)$. However, an important difference were found in CA scores $\left(F[1,49]=29.49, p<0.001, \eta_{p}^{2}=0.37\right)$, DL subscale (DL $=$ $\left.F[1,49]=13.0, p=0.001, \eta_{p}^{2}=0.21\right)$ as well as for total score $(F[1,49]=11.23, p=0.002)$. Taking together, these results demonstrate specific effects after the NR intervention. The difference between post and follow-up scores were significant, with a greater change in scores for the $\mathrm{CG}$, which means deterioration in executive functions, whereas the NRG scores remained similar to post-treatment.

Table 2

Sociodemographic and Clinical Characteristics for All Participants ${ }^{a}$

\begin{tabular}{lcc}
\hline \multirow{2}{*}{ Demographic and clinical variables } & NRG $(\mathrm{n}=20)$ & $\mathrm{CG}(\mathrm{n}=30)$ \\
\cline { 2 - 3 } & Mean (SD) or $\%$ & Mean (SD) or $\%$ \\
\hline Age (years) & $41.7(15.1)$ & $39.8(14.9)$ \\
Gender-\% Female & $72 \%$ & $69 \%$ \\
Education level (years) & $13.5(5.23)$ & $14.1(5.34)$ \\
Estimated IQ & $35.6(12.06)$ & $32.5(13.7)$ \\
BD types-\% Type I & $60 \%$ & $58 \%$ \\
Age at illness onset (years) & $23.4(7.48)$ & $26.6(8.54)$ \\
Number of episodes & $7(2.4)$ & $9(2.7)$ \\
Illness duration (years) & $24.8(13.5)$ & $22.6(14.7)$ \\
Number of hospitalizations & $3.72(2.7)$ & $2.98(2.2)$ \\
Pharmacological treatment: & & $69 \%$ \\
Mood stabilizers (lithium or valproate) and antipsychotics (risperidone, quetiapine) & $74 \%$ & $28 \%$ \\
Only mood stabilizers (lithium or valproate) & $25 \%$ & $3 \%$ \\
Only antipsychotics (risperidone, quetiapine) & $1 \%$ & \\
\hline
\end{tabular}

Notes. ${ }^{*}$ Estimated IQ based on Raven Score; ${ }^{\text {a }}$ No significant differences between groups.

\section{Ways of Coping}

The analyses for coping strategies focused on problems as well as for coping emotions subscale demonstrated a significant interaction between time measurements and type of treatment $\left(F[2,98]=11.12, p<0.001, \eta_{p}^{2}=\right.$ $0.19 ; F[2,98]=5.94, p=0.01, \eta_{p}^{2}=0.11$, respectively), which indicated that the performance over three assessments differed between the CG and NRG. The contrast to the difference between pre- and post-treatment when comparing the CG and NRG was also significant for both subscales (Problems $=F[1,49]=18.91, p<$ 0.001 , Emotions $\left.=F[1,49]=5.99, p=0.01, \eta_{p}^{2}=0.11\right)$. However, the contrast for post-treatment and follow-up was significant only for coping strategies focused on problems $(F[1,49]=4.78, p=0.03)$, whereas for coping strategies focused on emotions, there was no difference $\left(F[2,98]=2.03, p=0.14, \eta_{p}^{2}=0.04\right)$.

The main effect of time on the coping religiosity/fantasy thinking scores was not significant $(F[2,98]=$ $0.40, p=0.67, \eta_{p}^{2}=0.08$ ) indicating that there were no differences between the three measurements in different times (pre, post and follow up). Moreover, the results indicated that also there were no significant differences between groups $\left(F[1,49]=0.50, p=0.46, \eta_{p}^{2}=0.01\right)$ as well as no interaction between these two variables $\left(F[2,98]=1.03, p=0.36, \eta_{p}^{2}=0.02\right)$. 
Regarding coping strategies based on seeking social support, the main effect of time was not significant $\left(F[2,98]=1.37, p=0.26, \eta_{p}^{2}=0.03\right)$ which indicated that there were no significant differences between the three measurements taken at different times (pre, post and follow up). The presence of a significant main effect of treatment type $\left.F[1,49]=8.27, p<0.01, \eta_{p}^{2}=0.14\right)$ indicated an important difference between the two groups investigated. Finally, there was no significant interaction between measures and type of treatment $(F[2$, $98]=1.37, p=0.26, \eta_{p}^{2}=0.03$ ).

\section{WHOQOL-Bref}

It was observed a significant interaction between type measures time and treatment group only for physical health $\left(F[2,98]=6.14, p=0.004, \eta_{p}^{2}=0.11\right)$ and for psychological domains $(F[2,98]=10.39, p<$ $\left.0.001, \eta_{p}^{2}=0.18\right)$, indicating that the performance in these domains over the three assessments differed between the CG and NRG. There were no differences for the other domains: social relationships $(F[2,96]=$ $\left.1.47, p=0.24, \eta_{p}^{2}=0.03\right)$ and environmental $\left(F[2,96]=2.94, p=0.07, \eta_{p}^{2}=0.06\right)$.

Both contrast measures pre- $\times$ post-treament and post-treament $\times$ follow-up were statistically significant considering only the first two domains (Physical domain $=F[1,48]=8.22, p=0.006 ; F[1,48]=8.37, p=$ 0.006 and psychological domain $=F[1,48]=13.83, p=0.001 ; F[1,48]=15.54, p<0.001)$ respectively. Together these data show specific effects of the intervention was significant between groups.

\section{RCI}

Reliable change as defined by Jacobson and Truax (1991) was observed in a majority of the patients from NRG, ranging from $60 \%$ to $90 \%$ on DEX-R measures, from $60 \%$ to $80 \%$ on coping measures and from $45 \%$ to $65 \%$ on WHOQOL scores, considering pre- $\times$ post-treatment as well as pre-treatment $\times$ long-term six-month follow-up (see Table 3).

Table 3

Mean, SD, Effect Size, and Reliable Change Index at Pre-treatment, Post-treatment, and at Long-term Six-month Follow-up to Treatment

\begin{tabular}{|c|c|c|c|c|c|c|c|}
\hline $\mathrm{CG}^{\mathrm{a}}$ & $\begin{array}{l}\text { Pre-treatment } \\
(n=30)\end{array}$ & $\begin{array}{l}\text { Post-treatment } \\
(n=30)\end{array}$ & $\begin{array}{l}\text { Long-term } 6 \\
\text { months follow-up } \\
(n=30)\end{array}$ & $\mathrm{NRG}^{\mathrm{b}}$ & $\begin{array}{l}\text { Pre-treatment } \\
(n=20)\end{array}$ & $\begin{array}{l}\text { Post-treatment } \\
(n=20)\end{array}$ & $\begin{array}{l}\text { Long-term } 6 \\
\text { months follow-up } \\
(n=20)\end{array}$ \\
\hline \multicolumn{8}{|l|}{ DEX OFC $^{\mathrm{c}}$} \\
\hline Mean $(S D)$ & $34.13(13.33)$ & $37.19(10.16)$ & $34.97(8.12)$ & Mean $(S D)$ & $33.15(12.01)$ & $18.70(9.50)$ & $16.15(9.17)$ \\
\hline $\begin{array}{l}\text { Reliable } \\
\text { change, } n(\%)\end{array}$ & - & $2(6.6)$ & $1(3.33)$ & $\begin{array}{l}\text { Reliable } \\
\text { change, } n(\%)\end{array}$ & - & $17(85)$ & $14(70)$ \\
\hline \multicolumn{8}{|l|}{ DEX CA $^{\mathrm{d}}$} \\
\hline Mean $(S D)$ & $18,81(5,34)$ & $22.19(5.69)$ & $30.53(8.53)$ & Mean $(S D)$ & $21.60(6.18)$ & $12.40(6.69)$ & $11.20(6.00)$ \\
\hline $\begin{array}{l}\text { Reliable } \\
\text { change, } n(\%)\end{array}$ & - & $7(23.3)$ & $6(20)$ & $\begin{array}{l}\text { Reliable } \\
\text { change, } n(\%)\end{array}$ & - & $18(90)$ & $18(90)$ \\
\hline \multicolumn{8}{|l|}{$\mathrm{DEX} \mathrm{DL}^{\mathrm{e}}$} \\
\hline Mean $(S D)$ & $30.71(10.04)$ & $22.74(4.86)$ & $28.35(6.21)$ & Mean $(S D)$ & $23.30(6.47)$ & $14.10(6.72)$ & $12.65(6.20)$ \\
\hline $\begin{array}{l}\text { Reliable } \\
\text { change, } n(\%)\end{array}$ & - & $7(23.3)$ & $2(6.6)$ & $\begin{array}{l}\text { Reliable } \\
\text { change, } n(\%)\end{array}$ & - & $14(70)$ & $12(60)$ \\
\hline \multicolumn{8}{|c|}{ DEX TOTAL SCORE } \\
\hline Mean $(S D)$ & $81.97(16)$ & 90.77 (17.89) & $93.87(15.10)$ & Mean $(S D)$ & $78.05(20.21)$ & $45.20(20.41)$ & 40.00 (19.70) \\
\hline $\begin{array}{l}\text { Reliable } \\
\text { change, } n(\%)\end{array}$ & - & $1(3.33)$ & $4(13.33)$ & $\begin{array}{l}\text { Reliable } \\
\text { change, } n(\%)\end{array}$ & - & $18(90)$ & $18(90)$ \\
\hline
\end{tabular}


Table 3 continued

\begin{tabular}{|c|c|c|c|c|c|c|c|}
\hline $\mathrm{CG}^{\mathrm{a}}$ & $\begin{array}{l}\text { Pre-treatment } \\
(n=30)\end{array}$ & $\begin{array}{l}\text { Post-treatment } \\
(n=30)\end{array}$ & $\begin{array}{l}\text { Long-term } 6 \\
\text { months follow-up } \\
(n=30)\end{array}$ & $\mathrm{NRG}^{\mathrm{b}}$ & $\begin{array}{l}\text { Pre-treatment } \\
(n=20)\end{array}$ & $\begin{array}{l}\text { Post-treatment } \\
(n=20)\end{array}$ & $\begin{array}{l}\text { Long-term } 6 \\
\text { months follow-up } \\
(n=20)\end{array}$ \\
\hline \multicolumn{8}{|c|}{ COPING PROBLEMS $^{\mathrm{f}}$} \\
\hline Mean $(S D)$ & $3.09(0.66)$ & $2.74(0.66)$ & $3.21(0.67)$ & Mean $(S D)$ & $3.07(0.75)$ & $3.67(0.59)$ & $3.79(0.61)$ \\
\hline $\begin{array}{l}\text { Reliable } \\
\text { change, } n(\%)\end{array}$ & - & $4(13.33)$ & $2(6.66)$ & $\begin{array}{l}\text { Reliable } \\
\text { change, } n(\%)\end{array}$ & - & $13(65)$ & $13(65)$ \\
\hline \multicolumn{8}{|c|}{ COPING EMOTIONS ${ }^{\mathrm{g}}$} \\
\hline Mean $(S D)$ & $2.76(0.95)$ & $2.80(0.78)$ & $2.37(1.35)$ & Mean $(S D)$ & $3.06(0.70)$ & $2.44(0.70)$ & $2.34(0.67)$ \\
\hline $\begin{array}{l}\text { Reliable } \\
\text { change, } n(\%)\end{array}$ & - & $1(3.33)$ & $3(10)$ & $\begin{array}{l}\text { Reliable } \\
\text { change, } n(\%)\end{array}$ & - & $16(80)$ & $12(60)$ \\
\hline \multicolumn{8}{|c|}{ COPING RELIGIOSITY / FANTASY THINKINGh } \\
\hline Mean $(S D)$ & $3.19(1.28)$ & $2.84(1.28)$ & $3.23(1.13)$ & Mean $(S D)$ & $3.15(0.67)$ & $3.31(0.63)$ & $3.26(0.71)$ \\
\hline $\begin{array}{l}\text { Reliable } \\
\text { change, } n(\%)\end{array}$ & - & $4(13.33)$ & $2(6.66)$ & $\begin{array}{l}\text { Reliable } \\
\text { change, } n(\%)\end{array}$ & - & $5(25)$ & $3(15)$ \\
\hline \multicolumn{8}{|c|}{ COPING SOCIAL SUPPORT $^{\mathrm{i}}$} \\
\hline Mean $(S D)$ & $2.94(1.18)$ & $2.75(0.84)$ & $2.75(0.84)$ & Mean $(S D)$ & $3.35(0.54)$ & $3.58(0.72)$ & $3.59(0.72)$ \\
\hline $\begin{array}{l}\text { Reliable } \\
\text { change, } n(\%)\end{array}$ & - & $2(6.66)$ & $4(13.33)$ & $\begin{array}{l}\text { Reliable } \\
\text { change, } n(\%)\end{array}$ & - & $2(10)$ & $2(10)$ \\
\hline \multicolumn{8}{|c|}{ WHOQOL PHYSICAL HEALTH ${ }^{j}$} \\
\hline Mean $(S D)$ & $11.89(2.95)$ & $11.43(3.12)$ & $12.55(3.43)$ & Mean $(S D)$ & $11.40(2.68)$ & $14.16(2.52)$ & $11.09(2.39)$ \\
\hline $\begin{array}{l}\text { Reliable } \\
\text { change, } n(\%)\end{array}$ & - & $3(10)$ & $2(6.66)$ & $\begin{array}{l}\text { Reliable } \\
\text { change, } n(\%)\end{array}$ & - & $11(55)$ & $9(45)$ \\
\hline \multicolumn{8}{|c|}{ WHOQOL PSYCHOLOGICAL ${ }^{\mathrm{k}}$} \\
\hline Mean $(S D)$ & $11.69(3.33)$ & $10.70(2.83)$ & $12.81(3.74)$ & Mean $(S D)$ & $11.00(2.98)$ & $14.19(2.31)$ & $10.70(2.91)$ \\
\hline $\begin{array}{l}\text { Reliable } \\
\text { change, } n(\%)\end{array}$ & - & $2(6.66)$ & $4(13.33)$ & $\begin{array}{l}\text { Reliable } \\
\text { change, } n(\%)\end{array}$ & - & $13(65)$ & $10(50)$ \\
\hline \multicolumn{8}{|c|}{ WHOQOL SOCIAL $^{1}$} \\
\hline Mean $(S D)$ & $12.89(3.14)$ & $12.67(3.39)$ & $13.03(4.34)$ & Mean $(S D)$ & $11.07(3.99)$ & $13.33(2.70)$ & $12.27(3.37)$ \\
\hline $\begin{array}{l}\text { Reliable } \\
\text { change, } n(\%)\end{array}$ & - & $4(13.33)$ & $3(10)$ & $\begin{array}{l}\text { Reliable } \\
\text { change, } n(\%)\end{array}$ & - & $6(30)$ & $4(20)$ \\
\hline \multicolumn{8}{|c|}{ WHOQOL ENVIRONMENTAL $^{\mathrm{m}}$} \\
\hline Mean $(S D)$ & $12.00(2.50)$ & $12.52(2.20)$ & $13.05(2.50)$ & Mean $(S D)$ & $11.38(2.12)$ & $13.38(1.76)$ & $11.70(2.37)$ \\
\hline $\begin{array}{l}\text { Reliable } \\
\text { change, } n(\%)\end{array}$ & & $3(10)$ & $2(6.66)$ & $\begin{array}{l}\text { Reliable } \\
\text { change, } n(\%)\end{array}$ & - & $4(20)$ & $3(15)$ \\
\hline \multicolumn{8}{|c|}{ WHOQOL TOTAL SCORE } \\
\hline Mean $(S D)$ & $12.40(3.08)$ & $11.80(3.50)$ & $13.10(3.68)$ & Mean $(S D)$ & $11.40(3.95)$ & $13.40(3.84)$ & $12.70(3.45)$ \\
\hline $\begin{array}{l}\text { Reliable } \\
\text { change, } n(\%)\end{array}$ & & $6(20)$ & $5(16.66)$ & $\begin{array}{l}\text { Reliable } \\
\text { change, } n(\%)\end{array}$ & - & $13(65)$ & $11(55)$ \\
\hline \multicolumn{8}{|c|}{ 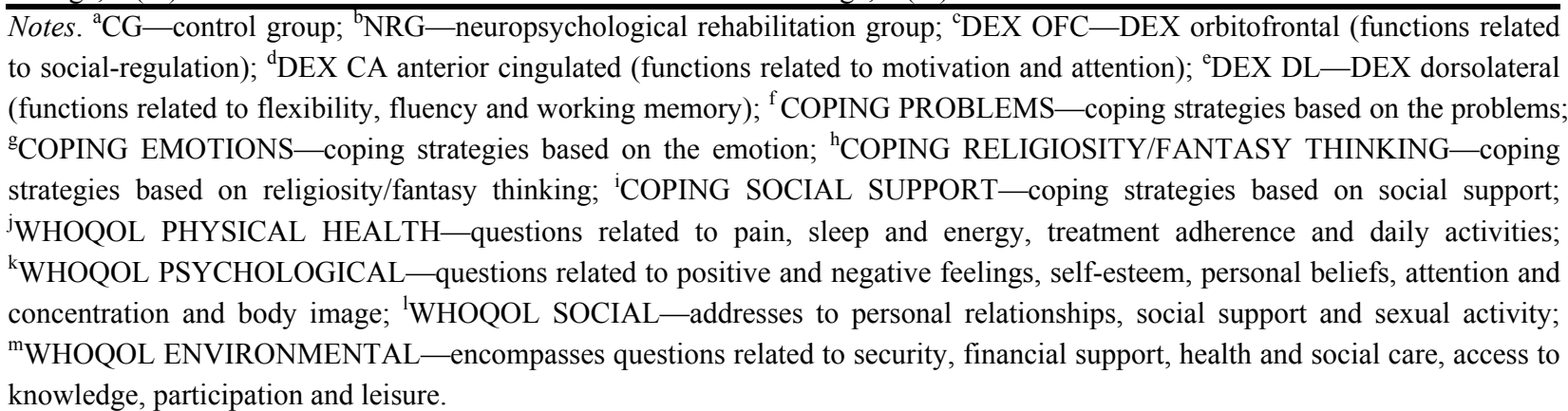 } \\
\hline
\end{tabular}




\section{Effects Sizes in Efficacy Measures}

Figure 2 displays the effects sizes (Cohen's $d$ ) (Cohen, 1988) calculated for the differences between the mean scores of pre- and post-treatment for the two groups of interest. In general, the effects size was very robust for the NRG $(d>0.80)$.

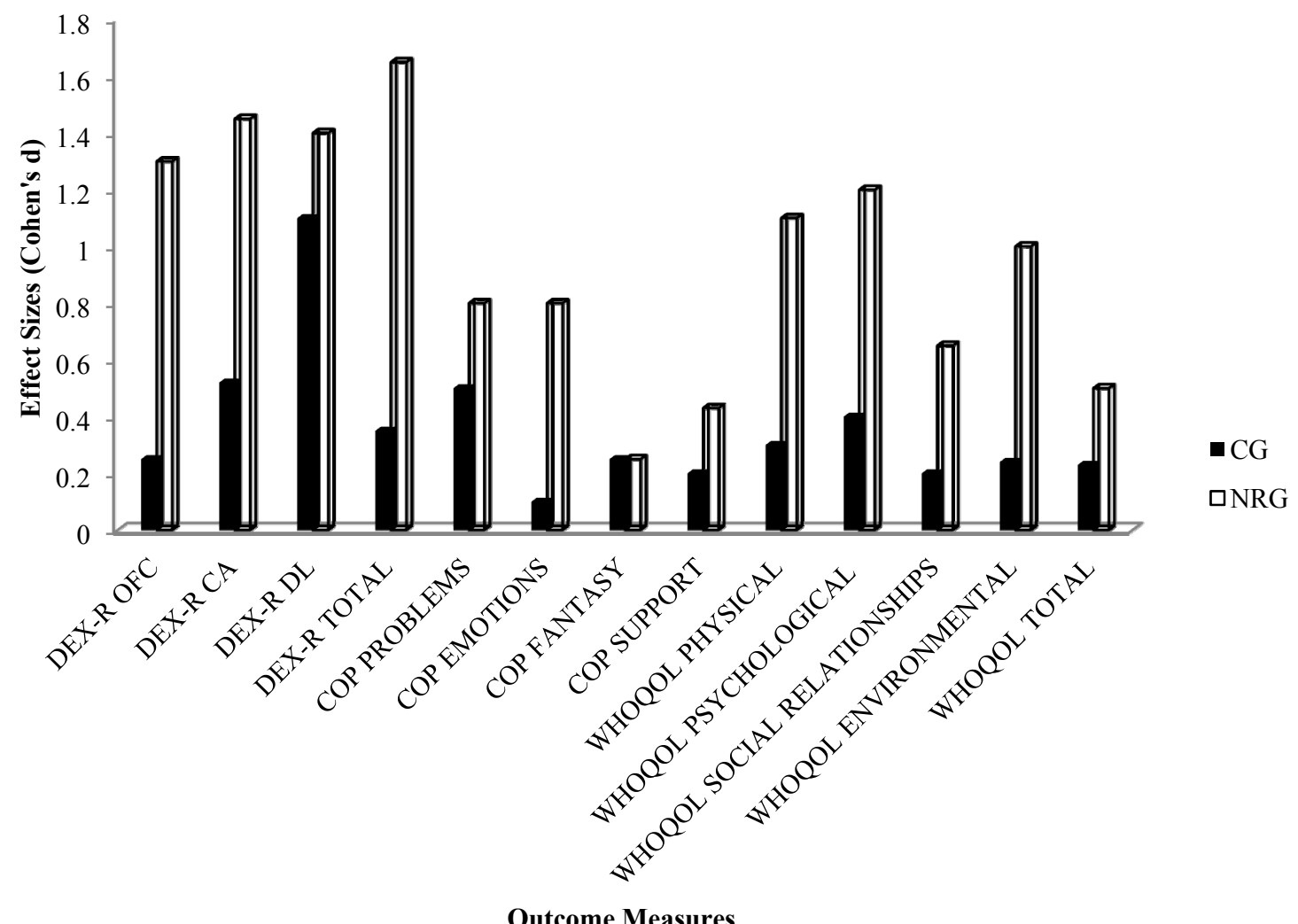

Figure 2. Effects sizes between groups for DEX-R, Whoqol-Bref and Ways of Coping Scale and respective domains.

\section{T-test}

The differences between groups comparison regarding the average of mood swings episodes are displayed in Table 4.

Table 4

Comparisons of Clinical Variables

\begin{tabular}{lll}
\hline Groups & Number of mood swings episodes & \\
\hline & Mean $(S D)$ & $t$-test $/ p$ \\
\hline CG & $3.3(1.7)$ & $t=5.823 / p<0.05$ \\
\hline
\end{tabular}

\section{Discussion}

In this study, we set out to investigate the efficacy of neuropsychological rehabilitation for bipolar patients. It was hypothesized that pharmacotherapy plus NR intervention would enhance the patient's functionality, which comprises in this study, a better performance on an ecological executive functions scale, an improvement 
in quality of life measures as well as the use of more adaptive coping strategies, i.e., strategies focused on problems instead of focused on emotions. The results presented above show significantly greater improvements for the NRG, highlighting the intervention's efficacy, and its superiority as well. At the completion of the NR protocol, the NRG exhibited a greater effect size within the range of the most pharmacotherapies and psychosocial therapies. Our results support the feasibility of broad, multi-domain NR intervention, focused on functional demands, for patients with BD. Firstly, we will comment the results in general and afterwards we will focus on specific measures.

To our knowledge, until currently, there were only two studies, Deckersbach et al. (2010) and Torrent et al (2013), which have investigated the efficacy of a cognitive intervention program in bipolar patients. The former indicated that at the end of treatment, as well as at the three-month follow-up, patients showed lower residual depressive symptoms, increased occupational, as well as overall psychosocial functioning. Torrent et al (2013) reported significant functional improvements from baseline to endpoint over the 21 weeks of treatment. However, in this investigation it was not considered the long-term outcome. Considering our results, it was in line with these studies. We have found a greater improvement in all functional measures, with specific gains in quality of life indicators as well as in more advantageous coping styles, which are more focused on problems rather than on emotions. And these results were sustained even though the suspension of NR protocol for many of the measures adopted, i.e., DL and CA DEX-R subscales and total DEX-R score, coping strategies focused on problems as well as for the physical and psychological domains of WHOQOL-Bref. Then more than the expected improvement at the endpoint of intervention, our investigation has demonstrated the positive long-term outcome as a consequence of NR treatment, which was a fundamental strength of it.

As pointed above, repeated measures analysis revealed significant functional improvement from baseline to endpoint for all DEX-R measures, for coping specific subscales which were coping strategies focused on problems as well as emotions, and also for physical and psychological domains from WHOQOL-Bref. For the others measures (coping strategies focused on religiosity/fantasy thinking and seeking social support and social relationships and environmental domains from WHOQOL-Bref) in which we have not found differences, it was expected, since they represent a more broad constructs, that goes beyond the reach of NR strategies, i.e., faith and religiosity, availability of financial resources, transportation. Similar great results were also found in comparisons between post-treatment and follow-up measures, except for OFC DEX-R subscale and coping focused on emotions. Specifically about OFC subscale, even though the average and standard deviationd were different, the groups have shown the same pattern and because of that the test has not detected the difference. However, clinically, their behavior can be considered distinct. Regarding coping emotions, we have not found any differences between groups at the follow-up.

Beyond the results presented above, other important contribution of this study was the differences found in the clinical variables. Then, beyond the increment in functional skills as a result of NR protocol, we have found a decrease in frequency of mood swings episodes. These results were in line with results from Harvey (2011) and Lewandowski, Cohen, and Ongur (2011) that have demonstrated an intrinsic conexion between cognition and mood.

The study has also some limitations. Considering that the main objective of a neuropsychological rehabilitation intervention is a broad functional improvement, comprehended as beyond cognitive improvement, we have chosen to not consider the cognitive performance in purely neuropsychological tests. Our decision was laid on Torrent et al. (2013) research that has highlighted that even though the significant functional 
improvement, it was not found any statistical significance on these tests. We decided to comprise cognitive functions in a more contextualized way, through the functional questionnaires applied. Then our results have a more ecological meaning. Second, we did not have a control passive group for NR intervention, which should be a group that would receive pharmacotherapy and should have a placebo weekly intervention at our centre. However, future investigations are now being delineated, which means that we are working on the inclusion of that group to expand the power of our analyses.

As such, understand the positive effect of NR treatment in BD is the first step towards ensuring that patients have more successful treatment outcomes. This was the first study to date to examine the efficacy of NR applied for BD patients, through a standardized and at the same time tailored protocol, which has considered the six-month follow-up, and has had as a clinical aim at the functional improvement.

\section{References}

Amini, H., \& Sharifi, V. (2012). Quality of life in bipolar type I disorder in a one-year followup. In Depression Research and Treatment. doi: 10.1155/2012/860745

Amorim, P. (2000). Mini International Neuropsychiatric Interview (MINI): Validação de entrevista breve para diagnóstico de transtornos mentais. Revista Brasileira de Psiquiatria, 22, 106-115.

Bearden, C. E., Hoffman, K. M., \& Cannon, T. D. (2001). The neuropsychology and neuroanatomy of bipolar affective disorder: A critical review. Bipolar Disorder, 3, 106-150.

Beck, A. T., Steer, R. A., \& Garbin, M. G. J. (1988). Psychometric properties of the beck depression inventory twenty-five years of evaluation. Clin Psych Review, 8, 77-100.

Bennett, P. C., Ong, B., \& Ponsford, J. (2005). Measuring executive dysfunction in an acute rehabilitation setting: Using the dysexecutive questionnaire (DEX). J. Int. Neuropsychol Soc., 11, 376-385.

Bowie, C. R., Depp, C., McGrath, J. A., et al. (2010). Prediction of realworld functional disability in chronic mental disorders: A comparison of schizophrenia and bipolar disorder. Am. J. Psychiatry, 167, 1116-1124.

Cohen, J. (1988). Statistical power analysis for the behavioral sciences (2nd ed.). Hillsdale, N.J.: Erlbaum.

Conners, K.(2003). Continuous performance test performance in a normative epidemiological sample. J. Abnorm. Child Psychol., $31,555-562$.

Deckersbach, T., Nierenberg, A. A., Kessler, R., Luno, H. E., Ametrano, R. M., Sachs, G., Rauch, S. L., \& Dougherty, D. (2010). Cognitive rehabilitation for bipolar disorder: An open trial for employed patients with residual depressive symptoms. CNS Neuroscience \& Therapeutics, 16, 298-307.

Fleck, M. P. A., Louzada, S., Xavier, M., Chachamovich, E., Vieira, G., Santos, L., et al. (2000). Aplicação da versão em português do instrumento abreviado de avaliação da qualidade de vida "WHOQOL-Bref". Rev. Saúde Pública., 34, $178-183$.

Greenhouse, W. J., Meyer, B., \& Johnson, S. L. (2000). Coping and medication adherence in bipolar disorder. J. Affect Disord., 59, 237-241.

Grassi-Oliveira, R., Daruy-Filho, L., \& Brietzke, E. (2010). New perspectives on coping in bipolar disorder. Psychology \& Neuroscience, 3, 161-165.

Harvey, P. D. (2011). Mood symptoms, cognition, and everyday functioning in major depression, bipolar disorder, and schizophrenia. Innov. Clin. Neurosci., 8, 14-18.

Jacobson, N. S., \& Truax, P. (1991). Clinical significance: A statistical approach to defining meaningful change in psychotherapy research. J. Consult Clin Psychol., 59, 12-19.

Kapczinski, F., Vieta, E., Andreazza, A. C., Frey, B. N., Gomes, F. A., Tramontina, J., \& Post, R. M. (2008). Allostatic load in bipolar disorders: Implications for pathophysiology and treatment. Neurosci. Biobehav Rev., 32, 675-692.

Lazarus, R. S., \& Folkman, S. (1984). Stress appraisal and coping. New York: Springer.

Lewandowski, K. E., Cohen, B. M., \& Ongur, D. (2011). Evolution of neuropsychological dysfunction during the course of schizophrenia and bipolar disorder. In Psychol Med.. doi: 10.1017/S0033291710001042.

Loschiavo-Alvares, F. Q., Sediyama, C. Y. N., Vasconcelos, A. G., Neves, F. S., Corrêa, H., Malloy-Diniz, L., \& Bateman, A. (2013). Clinical application of DEX-R for patients with bipolar disorder type I and II. Clinical Neuropsychiatry, 10, 86-94. 
Loschiavo-Alvares, F. Q., Sediyama, C. Y. N., Neves, F. S., Corrêa, H., Malloy-Diniz, L. F., \& Bateman, A. (2013). Neuropsychological rehabilitation for bipolar disorder-A single case design. Translational Neuroscience, 4, 1-8.

Malloy-Diniz, L. F., Leite, W. B., Moraes, P. H. P., Correa, H., Bechara, A., \& Fuentes, D. (2008). Brazilian Portuguese version of Iowa gambling task: Transcultural adaptation and discriminant validity. Rev. Bras. Psiquiatr., 30, 144-148.

Malloy-Diniz, L. F., Lasmar, V. A. P., Gazinelli, L. S. R., Fuentes, D., \& Salgado, J. V. (2007). The Rey auditory-verbal learning test: Applicability for the Brazilian elderly population. Rev. Bras. Psiquiatr., 29, 324-329.

Martinez-Arán, A., Vieta, E., Torrent, C., Sanchez-Moreno, J., Goikolea, J. M., Salamero, M., et al. (2007). Functional outcome in bipolar disorder: The role of clinical and cognitive factors. Bipolar Disord., 9, 103-113.

Matrinez-Aran, A., Torrent, C., Solé, B., Bonnín, M., Rosa, A. R., Sanchéz-Moreno, J., \& Vieta, E. (2011). Functional remediation for bipolar disorder. Clin Pract Epidemiol Ment Health, 7, 112-116.

Nolen-Hoeksema, S. (2000). The role of rumination in depressive disorders and mixed anxiety/depressive symptoms. J. Abnorm Psychol., 109, 504-511.

Perez, E. J. P., Leon, J. M. R. S., Mota, G. R., Luque, M. L., Arroyo, A. O., Saiz, J. C., \& Garcia, C. P. (2009). Spanish version of the dysexecutive questionnaire (DEX-Sp): Psychometric properties in addicts and non-clinical sample. Adicciones, 1, 155-166.

Robinson, L. J., Thompson, J. M., Gallagher, P., Goswami, U., Young, A. H., \& Ferrier, I. N. (2006). A meta-analysis of cognitive deficits in euthymic patients with bipolar disorder. J. Affect Disord., 93, 105-115.

Robinson, L. J., \& Ferrier, I. N. (2006). Evolution of cognitive impairment in bipolar disorder: A systematic review of cross-sectional evidence. Bipolar Disord., 8, 103-116.

Seidl, E. M. F., Tróccoli, B. T., \& Zannon, C. M. L. M. (2001). Análise fatorial de uma medida de estratégias de enfrentamento. Psicologia: Teoria e Pesquisa, 17, 225-234.

Simblett, S. K., \& Bateman, A. (2011). Dimensions of the dysexecutive (DEX) questionnaire examined using Rasch analysis. Neuropsychol Rehabil., 21, 1-25.

Sohlberg, M., \& Mateer, C. A. (2001). Cognitive rehabilitation: An integrative neuropsychological approach. New York: The Guildford Press.

The WHOQOL Group. (2000). Development of the World Health Organization WHOQOL-Bref quality of life assessment. Psychol Med., 28, 551-558.

Torrent, C., Bonnin, C. M., Martinez-Aran, A., et al. (2013). Efficacy of functional remediation in bipolar disorder: A multicenter randomized controlled study. Am. J. Psychiatry., AiA, 1-8.

Troster, A. I., Woods, S. P., \& Morgan, E. E. (2007). Assessing cognitive change in Parkinson's disease: Development of practice effect-corrected reliable change indices. Arch Clin Neuropsychol., 22, 711-718.

Wilson, B. (2005). Neuropsychological rehabilitation: Theory and practice. Lisse: Swits \& Zeitlinger.

Wilson, B. A., Alderman, N., Burgess, P. W., Emslie, H., \& Evans, J. J. (1996). Behavioural assessment of the dysexecutive syndrome. Bury St. Edmunds, UK: Thames Valley Test Company.

Young, R. C., Biggs, J. T., Ziegler, V. E., \& Meyer, D. A. (1978). A rating scale for mania: Reliability, validity and sensitivity. Br. J. Psychiatry, 133, 429-435. 